\title{
Valoración artroscópica de cartílago articular de cadera posterior a efecto de cutout de clavo centromedular de fémur
}

\section{Hip cartilage arthroscopic evaluation after cephalomedullary nail cutout}

\author{
Jorge Luis Martínez-Peniche, ${ }^{*}$ José Abonce-Zenteno, ${ }^{\ddagger}$ \\ Jorge Andrés Ávila-Del Pino, ${ }^{\ddagger}$ Pablo Felipe Tarazona-Velutini ${ }^{\ddagger}$
}

Citar como: Martínez-Peniche JL, Abonce-Zenteno J, Ávila-Del Pino JA, Tarazona-Velutini PF. Valoración artroscópica de cartílago articular de cadera posterior a efecto de cutout de clavo centromedular de fémur. An Med ABC. 2021; 66 (2): 132-136. https://dx.doi. org/10.35366/100483

\section{RESUMEN}

El fenómeno de cutout consiste en la protrusión del material de osteosíntesis en el cuello y cabeza femoral hacia la articulación de la cadera. Este suceso daña el cartílago articular de la cadera y cobra especial importancia por sus secuelas funcionales a largo plazo. El tratamiento consiste en el retiro del material de osteosíntesis y la evaluación del cartílago articular. Existen algoritmos de tratamiento para el daño condral en la cadera, dependiendo de la extensión y gravedad del daño. Presentamos el caso de un masculino de 28 años con efecto de cutout posterior a la fijación de una fractura diafisaria de fémur con clavo centromedular, y una nueva manera de examinar artroscópicamente el cartílago articular a través de los orificios dejados por el clavo centromedular.

Palabras clave: Artroscopia, cartílago articular, cadera, clavo centromedular.

\section{ABSTRACT}

The cutout phenomenon consists in the protrusion of osteosynthesis material through the femoral neck and head, putting the material in direct contact with the hip joint cartilage. This event damages the articular cartilage and has important implications in long term function. Standard treatment consists in removal of the material and direct evaluation of the articular cartilage. Different treatment algorithms exist, considering the extension and degree of chondral damage. We present the case of a 28-year-old male, with hip cutout injury posterior to surgical fixation of a diaphyseal femoral fracture with a cephalomedullary nail, and a new way of exploring arthroscopically the joint, using the orifices left by the removed implant.

Keywords: Arthroscopy, joint cartilage, hip, bone nails.

\footnotetext{
* Médico Residente.

‡ Médico de Staff.
}

Ortopedia y Traumatología. Centro Médico ABC. México.

Recibido: 11/04/2021. Aceptado: 01/06/2021.

Correspondencia:

Jorge Luis Martínez-Peniche

E-mail: jorchmartinez@gmail.com

\section{INTRODUCCIÓN}

La incidencia de las fracturas de fémur, comúnmente encontradas por el cirujano ortopedista, se estima en 10 fracturas por cada 100,000 habitantes. ${ }^{1}$ Su proporción por género es de 1.74 a uno con preferencia por el sexo masculino. Los hombres tienen un pico de incidencia entre los 15 y 24 años de edad; las mujeres en promedio a los 85 años. Estas lesiones se asocian usualmente a trauma de alta energía en el sexo 
masculino y a trauma de baja energía en las mujeres adultas mayores. ${ }^{2}$

El tratamiento considerado actualmente el estándar de oro para estas fracturas es el enclavado centromedular, el cual reduce su tasa de complicaciones. ${ }^{3,4}$ Esta modalidad de tratamiento permite movilidad temprana del paciente y consolidación ósea predecible.

El tratamiento con clavo centromedular de cadera no está exento de complicaciones; la más común es el efecto cutout o protrusión del material de osteosíntesis a través de la cabeza femoral. ${ }^{5}$ Se estima que el cutout femoral constituye $84 \%$ de las fallas en la fijación y ocurre hasta en $8 \%$ de los casos de enclavamiento centromedular. ${ }^{6}$ Los principales factores de riesgo de esta complicación son: mala calidad ósea, mala reducción de los fragmentos y el posicionamiento del tornillo cefálico en las porciones superolaterales y superocentrales del cuello femoral según la clasificación de Ikuta. ${ }^{5}$

La literatura enfocada en el tratamiento del $\mathrm{cu}$ tout recomienda predominantemente la revisión de la osteosíntesis, ya sea con reducción y fijación anatómica, o con osteotomías del fémur proximal. ${ }^{7}$ La protrusión del material de osteosíntesis puede dañar el cartílago articular al encontrarse en contacto directo con las superficies recubiertas de cartílago. ${ }^{8}$

El tratamiento de las lesiones cartilaginosas de la cadera debe personalizarse en función del tamaño de la lesión, su morfología y su localización dentro de la cadera. La artroscopia diagnóstica con desbridamiento artroscópico de la lesión es el tratamiento

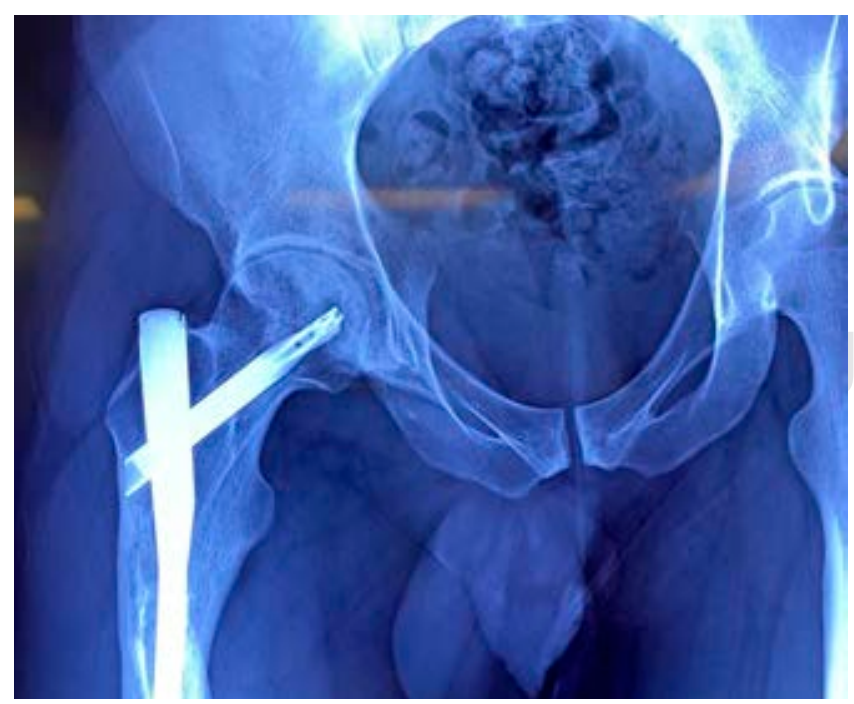

Figura 1: Radiografía anteroposterior de cadera derecha.

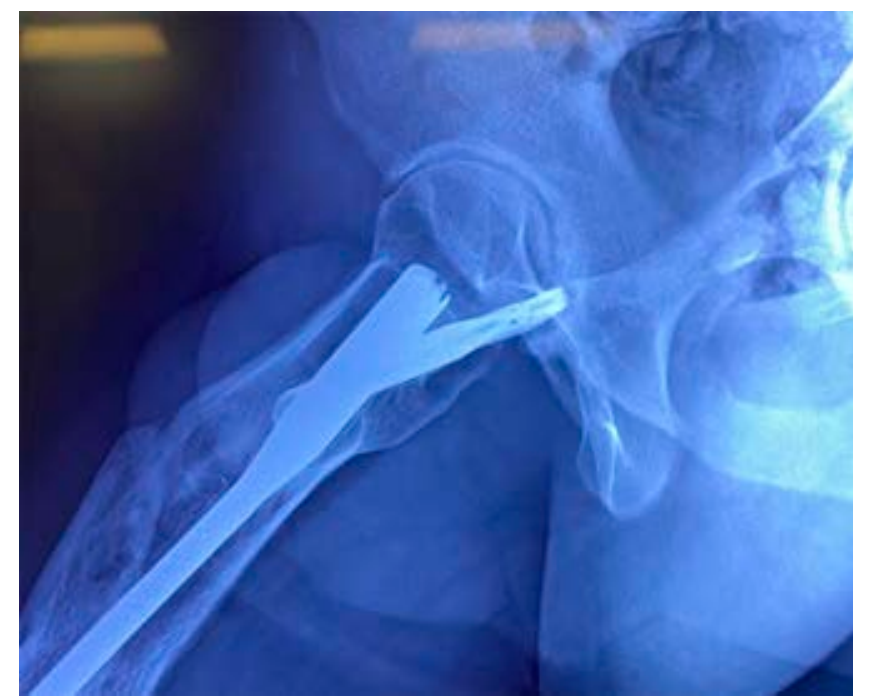

Figura 2: Radiografía lateral de cadera derecha.

más comúnmente utilizado hasta en $49 \%$ de los casos reportados. ${ }^{9}$

\section{PRESENTACIÓN DEL CASO}

Se trata de un hombre de 28 años con antecedente de fractura diafisaria de fémur derecho tratada mediante enclavado centromedular en septiembre de 2018. Durante su periodo postoperatorio continúa con limitación en los arcos de movilidad de la cadera y la examinación radiográfica evidenció retraso en la consolidación, por lo que se decide dinamización del implante en julio de 2019 mediante retiro del tornillo distal.

Posterior a la dinamización del clavo centromedular, el paciente continúa con dolor referido a cadera derecha, disminución de los arcos de movimiento e incapacidad para la bipedestación mayor de 30 minutos. Acude a valoración donde se encuentra con acortamiento de $2 \mathrm{~cm}$ y actitud en rotación externa en el miembro pélvico derecho. Los arcos de movilidad de la cadera derecha se encuentran con $60^{\circ}$ a la flexión activa y pasiva, $20^{\circ}$ de extensión, abducción de $40^{\circ}$, aducción de $15^{\circ}$, rotación externa a $5^{\circ}$ y rotación externa $5^{\circ}$. La flexión se encontraba limitada por dolor. Las pruebas específicas de provocación FABER (dolor a la flexión, abducción y rotación externa), FADIR (dolor a la flexión, aducción y rotación externa) eran positivas. Fuerza 4/5 para flexión de cadera derecha limitada por dolor. Sensibilidad y reflejos conservados. La cadera izquierda se explora con arcos de movilidad completos y sin otras alteraciones. 
An Med ABC. 2021; 66 (2): 132-136

Las radiografías de cadera derecha en proyección anteroposterior (Figura 1) y lateral (Figura 2), así como radiografías de eje mecánico (Figura 3), evidenciando fractura consolidada de fémur diafisario derecho, acortamiento de $2.04 \mathrm{~cm}$ de miembro pélvico derecho a expensas de fémur y clavo centromedular anterógrado de entrada trocantérica. La radiografía lateral de cadera derecha revela protrusión posterior de la hélice del clavo centromedular a través de la cabeza femoral encontrándose en contacto con pared acetabular posterior. Este contacto condiciona erosión de pared acetabular alrededor de la protrusión.

Realizamos retiro del material de osteosíntesis de fémur derecho y artroscopia de cadera derecha. Durante la artroscopia se utilizaron portales anteriores y anterolateral estándar de visión; utilizamos túnel existente a través de cuello femoral donde se encontraba hélice cefálica para valorar superficie articular en el punto de protrusión del clavo.

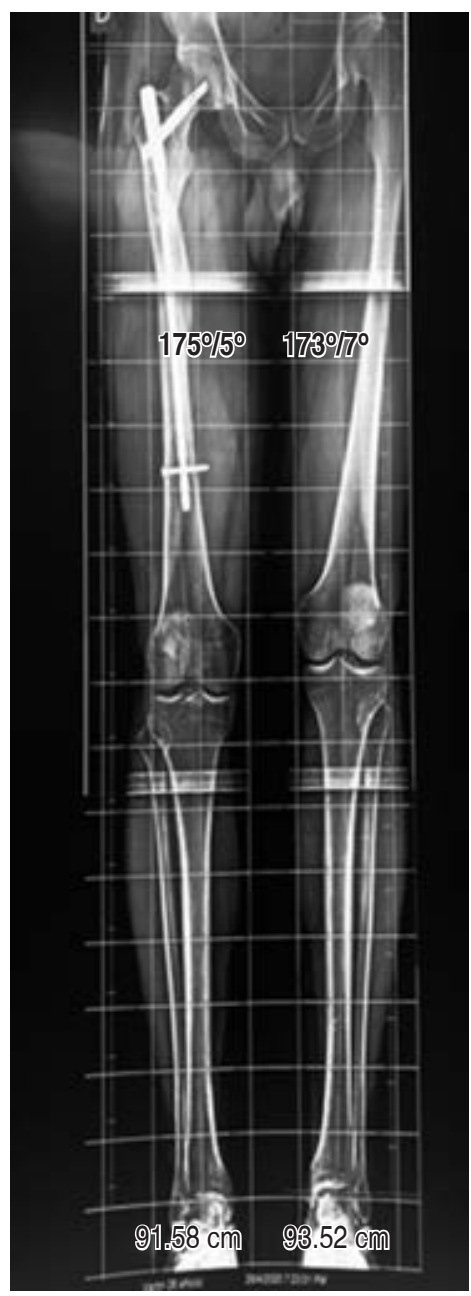

Figura 3:

Eje mecánico de miembros inferiores.

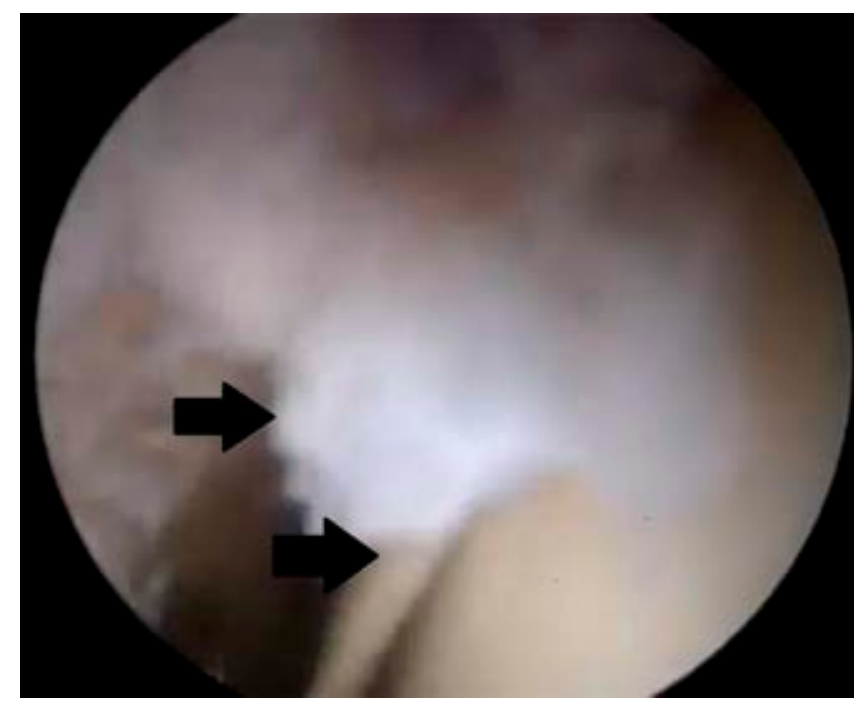

Figura 4: Visión artroscópica. Cuerpos libres intraarticulares (flechas).

A la examinación artroscópica observamos cuerpos libres intraarticulares y daño condral en superficie en contacto con material de osteosíntesis (Figura 4). A través de túnel transóseo documentamos protrusión de material de osteosíntesis y contacto con superficie articular coxofemoral. La examinación dinámica mediante movilización de cadera derecha nos permitió observar zonas de lesión condral grado 4 de Outerbridge en la superficie de contacto de hélice en acetábulo posteromedial (Figura 5). Las superficies desprovistas de cartílago desbridaron con rasurador eléctrico hasta obtener bordes estables. Posterior al desbridamiento se documentó una zona lesional de menos de $1 \mathrm{~cm}^{2}$, por lo que nuestro tratamiento fue la regularización de los bordes y la estabilización del cartílago restante.

Nuestro paciente evolucionó de manera favorable en su periodo postoperatorio. Su seguimiento a los seis meses después del retiro de material y la artroscopia de cadera mostraba arcos de movilidad de $80^{\circ}$ a la flexión, $20^{\circ}$ a la extensión, rotación interna $20^{\circ}$, rotación externa $40^{\circ}$, abducción $40^{\circ}$, aducción $15^{\circ}$ de la cadera intervenida. Sin dolor a la bipedestación, marcha a los arcos de movilidad. El paciente se reincorporó a su nivel previo de actividad recreativa y laboral.

\section{DISCUSIÓN}

En este paciente la prioridad posterior al diagnóstico de la protrusión del material de osteosíntesis es la preservación de la cadera nativa y el cartílago articular. Nuestra propuesta de tratamiento se encamina- 
ba en retirar prontamente el implante que lesiona al cartílago articular de la cadera, y tratar las secuelas del daño hecho por el mismo. Con base en lo anterior, propusimos al paciente retirar el material y examinar el cartílago articular por artroscopia.

La artroscopia de cadera constituye un método mínimamente invasivo para tratar la patología intraarticular de la misma. Tiene como ventaja evitar las luxaciones quirúrgicas de cadera que implican la capsulotomía extensa y el corte del ligamento redondo. ${ }^{10}$ Existen, sin embargo, limitaciones técnicas para la artroscopia de cadera como el peso del paciente y la necesidad de mesas de tracción y equipo especializado de artroscopia. Se tiene también una inclinada curva de aprendizaje para poder realizar estos procedimientos de manera efectiva. ${ }^{11}$

Dallich y colaboradores refieren en su artículo ${ }^{9}$ que el grado de lesión del cartílago articular es la guía que debe tomar el cirujano para tratar la lesión condral. Existen múltiples tratamientos recomendados, desde los más sencillos como el desbridamiento del cartílago dañado, hasta los más complejos como la implantación de condrocitos autólogos cultivados en dos tiempos quirúrgicos. El mismo artículo menciona un algoritmo de tratamiento en función del tamaño de la lesión y la actividad física del paciente. ${ }^{12}$

Nuestra principal innovación en este caso fue la utilización de las perforaciones creadas por el material de osteosíntesis hacia la cadera para examinar el cartílago articular. El implante utilizado en este paciente, el clavo centromedular TFN-Advanced (DePuy Synthes Companies, Raynham, MA, EE.UU.)

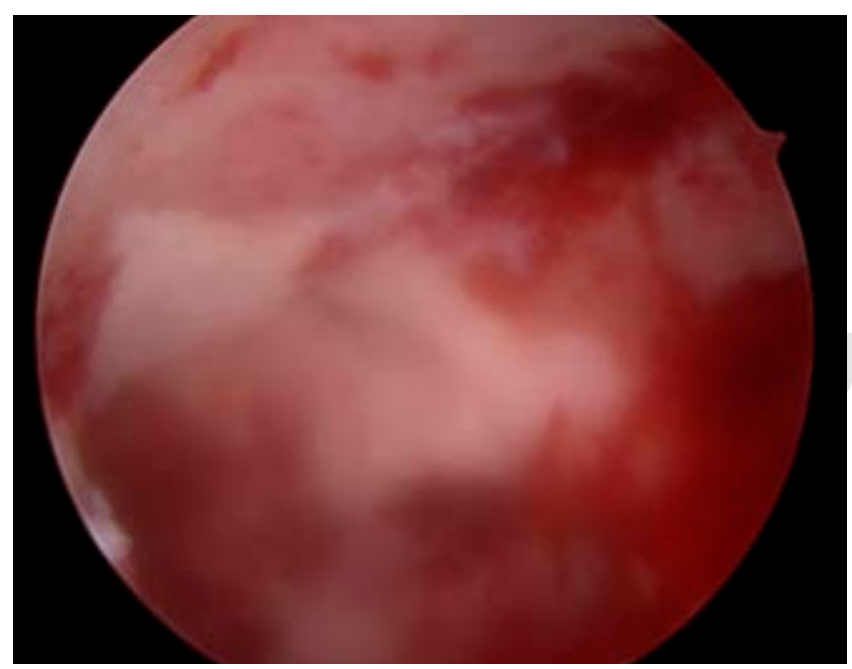

Figura 5: Visión a través de túnel óseo existente. Se aprecia daño condral grado 4 de Outerbridge, sangrado reciente y cuerpos libres. utiliza tornillos cefálicos de $10.35 \mathrm{~mm}$ de diámetro, ${ }^{13}$ lo cual nos permite fácilmente introducir un artroscopio estándar de $4 \mathrm{~mm}$ a través del túnel creado al quitar el material, y examinar directamente el daño articular a lo largo de todo el arco de movimiento. Este gesto quirúrgico, en conjunto con la técnica tradicional de artroscopia, nos permitió evaluar de manera integral el daño articular creado por el material de osteosíntesis protruyente.

Aunque se ha reportado el uso de artroscopia para el tratamiento de material protruyente dentro de la cadera, ${ }^{8}$ es del entendimiento de los autores que no se han utilizado los túneles del material protruyente dentro de la cadera como acceso para la visualización intraarticular. Este recurso puede ser útil para la primera visualización intraarticular debido a su orientación directa hacia la zona lesionada, aunque no permite la examinación de la superficie articular femoral, sólo la acetabular. De igual manera, este gesto no permite la evaluación de la porción cervical del fémur proximal, parte igualmente importante en la artroscopia diagnóstica de la cadera.

\section{CONCLUSIÓN}

Debemos recalcar la necesidad de un diagnóstico y tratamiento temprano para las lesiones condrales de cadera, especialmente en pacientes jóvenes. Su tratamiento oportuno permite la preservación de la cadera nativa. Ya existen protocolos y algoritmos para el tratamiento de las lesiones condrales en función de su tamaño y morfología. La artroscopia resulta valiosa en estos casos por ser mínimamente invasiva, y utilizar los túneles ya creados puede ser un recurso útil en pacientes a los cuales se les retiran implantes similares de manera concomitante a la exploración artroscópica.

\section{REFERENCIAS}

1. Weiss RJ, Montgomery SM, Al Dabbagh Z, Jansson KA. National data of 6409 Swedish inpatients with femoral shaft fractures: stable incidence between 1998 and 2004. Injury. 2009; 40 (3): 304-308.

2. Salminen ST, Pihlajamaki HK, Avikainen VJ, Bostman OM. Population based epidemiologic and morphologic study of femoral shaft fractures. Clin Orthop Relat Res. 2000; (372): 241-249.

3. Fakhry SM, Rutledge R, Dahners LE, Kessler D. Incidence, management, and outcome of femoral shaft fracture: a statewide population-based analysis of 2805 adult patients in a rural state. J Trauma. 1994; 37 (2): 255-260; discussion 260-261.

4. Rudloff MI, Smith WR. Intramedullary nailing of the femur: current concepts concerning reaming. J Orthop Trauma. 2009; 23 (5 Suppl): S12-S17. 
An Med ABC. 2021; 66 (2): 132-136

5. Turgut A, Kalenderer O, Karapinar L, Kumbaraci M, Akkan HA, Agus H. Which factor is most important for occurrence of cutout complications in patients treated with proximal femoral nail antirotation? Retrospective analysis of 298 patients. Arch Orthop Trauma Surg. 2016; 136 (5): 623-630.

6. Lorich DG, Geller DS, Nielson JH. Osteoporotic pertrochanteric hip fractures: management and current controversies. Instr Course Lect. 2004; 53: 441-454.

7. Wu CC, Shih CH, Chen WJ, Tai CL. Treatment of cutout of a lag screw of a dynamic hip screw in an intertrochanteric fracture. Arch Orthop Trauma Surg. 1998; 117 (4-5): 193196.

8. Jang JH, Moon NH, Park KY. Arthroscopic management of intraarticular screw perforation after surgical treatment of an acetabular posterior wall fracture: a case report. Hip Pelvis. 2018; 30 (1): 60-64.
9. Dallich AA, Rath E, Atzmon R, Radparvar JR, Fontana A, Sharfman $\mathrm{Z}$ et al. Chondral lesions in the hip: a review of relevant anatomy, imaging and treatment modalities. J Hip Preserv Surg. 2019; 6 (1): 3-15.

10. Briggs KK, Bolia IK. Hip arthroscopy: an evidence-based approach. Lancet. 2018; 391 (10136): 2189-2190.

11. Flecher X, Ollivier M, Parratte S. Artroscopia de cadera. EMC Técnicas Quirúrgicas en Ortopedia y Traumatología. 2018; 10 (3): 3-8.

12. Oliver-Welsh L, Griffin JW, Meyer MA, Gitelis ME, Cole BJ. Deciding how best to treat cartilage defects. Orthopedics. 2016; 39 (6): 343-350.

13. DePuy Synthes. TFN-ADVANCED ${ }^{\mathrm{rm}}$ Proximal Femoral Nailing System (TFNA). Surgical Technique. [Consultada 27/10/2020]. Disponible en: https://www.jnjmedicaldevices. com/en-EMEA/product/tfn-advanced-proximal-femoralnailing-system 